\title{
NRAS Expression is Associated With Prognosis and Tumor Immune Microenvironment in Lung Adenocarcinoma
}

\section{Yueren Yan}

Fudan University Shanghai Cancer Center

\section{Zhendong Gao}

Fudan University Shanghai Cancer Center

Han Han

Fudan University Shanghai Cancer Center

\section{Yue Zhao}

Fudan University Shanghai Cancer Center

\section{Yang Zhang}

Fudan University Shanghai Cancer Center

\section{Xiangyi Ma}

Fudan University Shanghai Cancer Center

haiquan Chen ( $\nabla$ hqchen1@yahoo.com )

Fudan University Shanghai Cancer Center https://orcid.org/0000-0002-1305-971X

\section{Research Article}

Keywords: NRAS, Lung adenocarcinoma, Prognosis, Tumor immune microenvironment

Posted Date: September 3rd, 2021

DOl: https://doi.org/10.21203/rs.3.rs-862652/v1

License: (9) This work is licensed under a Creative Commons Attribution 4.0 International License. Read Full License

Version of Record: A version of this preprint was published at Journal of Cancer Research and Clinical Oncology on November 8th, 2021. See the published version at https://doi.org/10.1007/s00432-02103842-w. 


\section{Abstract}

Purpose: NRAS plays a pivotal role in progression of various kinds of somatic malignancies; however, the correlation between NRAS and lung adenocarcinoma is less known. We aim to analyze the prognostic value of NRAS expression in lung adenocarcinoma, and explore the relationship between NRAS and tumor immune microenvironment.

Methods: We obtained the transcriptome pofiles and clinical data of LUAD from The Cancer Genome Atlas database and three Genome Expression Omnibus datasets. Specimens from 325 patients with completely resected lung adenocarcinoma were collected for immunohistochemical assays of NRAS, PDL1, PD-1 and TIM-3. Then we performed gene set enrichment analysis to investigate cancer-related and immune-related signaling pathways. TIMER algorithms were performed to evaluate tumor immune infiltrating cells and immune-related biomarkers.

Results: Compared with adjacent non-tumor tissue, NRAS expression was significantly upregulated in LUAD tissue. NRAS expression was significantly correlated with more advanced stage and positive lymph nodes. Kaplan-Meier curves and Cox analysis suggested that high NRAS expression led to a poor prognosis, and could be an independent prognostic factor in LUAD patients. Besides, NRAS expression was positively correlated with CD8+ T cells, macrophages, and neutrophils, and negatively correlated with $B$ cells and CD4+ T cells. The expression level of NRAS was positively correlated with PD-L1, PD-1, and TIM-3 both at RNA and protein level.

Conclusions: To conclude, we found NRAS a novel prognostic biomarker in LUAD. Besides, the expression level of NRAS may influence the prognosis of LUAD via various kinds of cancer-related pathways and remodeling TIM.

\section{Introduction}

Lung cancer remains the leading cause of cancer-related death and has the highest incidence rate worldwide. (Bray et al., 2018). Among all the histological subtypes of lung cancer, lung adenocarcinoma (LUAD) is the one which dominant, with an average 5-year survival rate of 15\%. (Siegel, Miller, \& Jemal, 2017) Over the past decades, advances in high throughput sequencing techniques have expanded our knowledge about gene expression and genetic alterations at the pan-genomic level in various somatic malignancies. Numerous large-scale studies have demonstrated that different genetic subtypes have distinctive survival rates in malignancies. (Liloglou, Bediaga, Brown, Field, \& Davies, 2014) (Vogelstein et al., 2013) Therefore, identifying genetic biomarkers were in great need to predict prognosis of LUAD.

Neuroblastoma rat sarcoma viral oncogene homolog (NRAS) was originally identified as the third RAS family member following KRAS and HRAS in human neuroblastoma and fibrosarcoma cell lines. (Shimizu et al., 1983) Akin to KRAS, NRAS encodes small GTP enzymes which regulate cell cycle, proliferation, maturation and differentiation by transducing signals from membrane-localized receptor tyrosine kinases to the nucleus. The mutation of RAS gene family occurs in nearly $30 \%$ of human 
cancers. (Downward, 2003) NRAS plays an important role in EGFR mediating RAS/RAF/MEK/ERK and $\mathrm{PI} 3 \mathrm{~K} / \mathrm{AKT} / \mathrm{mTOR}$ signaling pathways. (Whitwam et al., 2007) The mutation of NRAS leads to continuous activation of Ras-GTP, which promotes tumorigenesis and metastasis. It has been proved that NRAS acts as a negative prognosis predictor in numerous somatic malignancies, such as melanoma, colorectal cancer and hepatocellular carcinoma. (Banys-Paluchowski et al., 2020; Bertoli, Giavarra, Vitale, \& Minisini, 2019; Dietrich et al., 2019; Hu et al., 2018)

Besides, NRAS, as a proto-oncogene, is also proved to be associated with tumor immune microenvironment (TIM) both in vivo and in vitro. Castro et al. has found that NRAS GTPase is related to T cell proliferation and immune response. (Pérez de Castro et al., 2003)Thomas et al. indicates that NRAS mutation in advanced melanoma is correlated with increased benefit from immunotherapy. (Thomas et al., 2015) TIM has already been recognized as an essential factor in cancer progression. Its prognostic value has been proven in LUAD. (Brambilla et al., 2016; Suzuki et al., 2011) For instance, Brambilla et al. demonstrates that higher $C D 4+/ C D 8+$ ratio conferred a better survival of patients suffering from lung cancer. (Brambilla et al., 2016) Besides, tumor mutation burden and expression level of PD-L1 have been widely used to predict outcomes in lung cancer patients receiving immunotherapy.

Till now, there is only one multi-institutional study, which has initially explored the relationship between NRAS mutations and lung cancer. This report indicates that LUAD patients with NRAS mutations may have potential sensitivity to MEK inhibitors. (Ohashi et al., 2013)Unfortunately, the correlation between NRAS and LUAD is still unclear. Therefore, NRAS seems to be a logic alternative to investigate for potential novel biomarkers in LUAD.

In this study, we analyzed the prognostic value of NRAS in LUAD based on public databases and tumor microarrays of 325 patients with LUAD. Additionally, we explored the relationship between NRAS and tumor immune microenvironment both at RNA and protein level, together with potential molecular mechanisms of NRAS in LUAD.

\section{Methods}

\subsection{Tissue samples}

LUAD patient datasets, with transcriptome profile and clinical information, were obtained from the public online datasets TCGA-LUAD(http://portal.gdc.cancer.gov/), including 497 LUAD tumor tissues and 54 adjacent non-malignant lung tissues. Furthermore, three lung cancer chips GSE50081 (including 181 NSCLC tumor samples, GPL570 [HG-U133_Plus_2] Affymetrix Human Genome U133 Plus 2.0 Array), GSE42127 (176 lung cancer samples, GPL6884 Illumina HumanWG-6 v3.0 expression beadchip), and GSE30219 (293 lung cancer samples and 14 non tumor lung samples, GPL570 [HG-U133_Plus_2] Affymetrix Human Genome U133 Plus 2.0 Array) were downloaded from Gene Expression Omnibus (GEO) database (https://www.ncbi.nlm.nih.gov/geo/) for a valid confirmation. 
The corhort to perform immunohistochemical (IHC) tissue microarray (TMA) consisted of 325 LUAD patients from Fudan University Shanghai Cancer Center (FDUSCC) diagnosed between 2009 and 2015. All these samples were obtained after surgical resection from patients who had never received neoadjuvant therapy.

\subsection{IHC expression achieved from the Human Protein Atlas}

IHC staining images were achieved from the Human Protein Atlas (HPA) (http://www.proteinatlas.org/) in order to validate the expression of NRAS in LUAD and adjacent non-tumor tissues.(Uhlén et al., 2015) Both the tumor tissues and normal tissues were stained by Antibody CAB010157. The staining location was cytoplasmic and membranous. The direct links to these images are as follows:

LUAD tissue: https://www.proteinatlas.org/ENSG00000213281-NRAS/pathology/lung+cancer\#img

Normal lung: https://www.proteinatlas.org/ENSG00000213281-NRAS/tissue/lung\#img

\subsection{IHC staining and evaluation of IHC intensity}

Formalin-fxed parafn-embedded primary specimens of patients with LUAD were obtained to construct 4$\mu \mathrm{m}$-thick sections. IHC staining was performed as previously described. (Fu et al., 2021) Immunostaining analysis was independently evaluated by Yan and Gao who were to the experimental groups using immunoreactive score (IRS). The staining intensity was graded 4 levels (no staining $=0$, weak staining $=1$, moderate staining $=2$, and strong staining $=3$ ) and the staining extent was graded 5 levels (no $=0,<10 \%=1$, $10 \%-50 \%=2,51-80 \%=3$, and $>80 \%=4$ ). The scores of the staining intensity and extent were multiplied to give a final staining score of $0 \sim 12$. The cutoff for IRS of NRAS was 6 . Antibodies against the NRAS (ab77392, 1:500, Abcam), PD-L1 (E1L3N, 1:1000, Cell Signaling Technology), TIM-3(D5D5R, 1:1000, Cell Signaling Technology), and PD-1 (DW42J, 1:500, Cell Signaling Technology) were used.

\subsection{Correlation analysis between NRAS expression and immune cell infiltration in LUAD}

Tumor immune estimation resource (TIMER 2.0) (http://timer.cistrome.org/), a useful online tool for comprehensive analysis of tumor-infiltrating immune cells (TIICs), (Li et al., 2020) was used to evaluate the relationship between NRAS expression and TIICs in LUAD. The level of TIICs is an essential indicator reflecting TIM. In addition, we analyze the correlation between immune-related functional molecular and NRAS via TIMER website. The TIIC and immune-related molecular levels of LUAD patients from TCGA datasets were derived from TIMER website.

\subsection{Gene sets enrichment analysis (GSEA)}

A computational method that determines the statistical significance of a priori defined set of genes and the existence of concordant differences between two biological states is known as the GSEA. (Subramanian, Kuehn, Gould, Tamayo, \& Mesirov, 2007) In this study, GSEA 4.0.3 generated an 
initial gene list on the classification of the genes according to their correlation with the NRAS expression. For each analysis, we performed 1000 repetitions of gene set permutations. The phenotype label that we put forth was the expression level of NRAS. Additionally, to sort the enriched pathways in each phenotype, we utilized the nominal p-value and normalized enrichment score (NES) (Subramanian et al., 2005). Gene sets with false discovery rate (FDR) $<0.25$ and nominal $P$ value $<0.05$ were considered to be significantly enriched. The gene sets were enriched and analyzed by Hallmark (h.all.v6.2.symbols.gmt) and Go (c5.bp.v6.2.symbols.gmt) Collection.

\subsection{Statistical Analysis}

Statistical analysis was performed using R-3.6.1. (R Foundation for Statistical Computing, Vienna, Austria) Group comparison analyses with continuous variables were evaluated by Wilcox $T$ test. The correlation between expression of NRAS and clinical features was performed with logistic analysis. Kaplan-Meier survival curves and log-rank test were applied to compare survival outcomes. Finally, univariate and multivariable Cox analyses were used to identify independent prognostic predictors. A statistical difference was taken as two-sided $P$ value $<0.05$.

\section{Results}

\subsection{Demographics of population in TCGA LUAD datasets}

497 LUAD patients with both clinical and transcriptome profiling were downloaded from TCGA LUAD datasets. After excluding those with missing or insufficient data on age, gender or stage, we included 316 patients to further examine the prognostic role by Cox analysis and Logistic regression. We also included 325 LUAD patients from FDUSCC diagnosed between 2009 and 2015 to perform IHC microarrays. The baseline characteristics of these two cohorts were presented in Table 1.

Correlations between the clinical information and NRAS were analyzed by logistic analyses in TCGA cohort. The categorical dependent value is based on the median value NRAS expression. As shown in Table2, high expression of NRAS in LUAD was significantly associated with advanced stage (stage I vs stage III, $\mathrm{OR}=1.90(1.14-3.21), \mathrm{p}=0.0059)$ and positive lymph nodes (positive vs negative, $\mathrm{OR}=2.00(1.26$ 3.20), $p=0.0037)$.

\subsection{NRAS expression is up-regulated in LUAD specimens}

Wilcox T test was performed to estimate the relationship between NRAS expression and disease status of LUAD based on TCGA datasets. As shown in Fig. 1 A, the expression of NRAS was significantly upregulated in LUAD samples compared with normal samples $(\mathrm{p}=3.85 \mathrm{e}-04)$. Meanwhile, we paired the tumor tissue and adjacent tumor tissue of the same patient from TCGA LUAD datasets. The pair analysis shown in Fig. 1B indicated the same results as the former ( $p=5.19 \mathrm{e}-05) .11$ LUAD tissue and 3 normal tissue IHC images were obtained from the HPA website. The staining intensity of LUAD tissue was medium-strong ( 2 of 11 were strong), while that of normal pneumocytes was weak. As shown in Fig.1C 
and Fig.1E (intensity: strong, staining: high, quantity $>75 \%$ ), the tumor cells were intensely stained. As shown in Fig. 1D and Fig. 1F (Macrophage: Intensity: medium, staining: moderate, quantity: $>75 \%$; Pneumocyte: Intensity: weak, staining: low, quantity: $75 \%-25 \%)$, the intensity of macrophage staining is moderate, and that of pneumocytes is weak in normal tissues. This indicates that NRAS acts as a pivotal role in LUAD progression.

\subsection{NRAS expression predicted survival and could be used as an independent prognostic biomarker in LUAD patients}

Patients were divided into high expression group and low expression group according to the median value of NRAS expression. As shown in Fig. 2A, Kaplan-Meier survival analysis shows that high NRAS expression group has a significantly poorer overall survival (OS) compared with low expression group. The univariable analysis shown in Table3a reveals that NRAS expression correlated significantly with a poor OS. Besides, clinical stage, tumor size and lymph nodes are significantly associated with OS. According to multivariable analysis (Table3b), the expression level of NRAS remains independently associated with OS $(p=0.012)$. Besides, we verified the prognostic value of NRAS at the protein level. We evaluated the protein level of NRAS in completely resected tissues using TMA. A total of 325 LUAD patients, undergoing surgical resection in Fudan University Shanghai Cancer Center, were enrolled in this study. According to the IRS of IHC staining, we defined 0-6 as weak staining group, and 7-12 as high staining group. The representative IHC images of NRAS are shown in Fig. 2D. As shown in Fig. 2C, higher expression of NRAS is also associated with worse OS at the protein level $(p=0.0072)$.

The prognostic value of NRAS in LUAD was also verified by three GEO datasets GSE50081 (including 181 LUAD samples), GSE42127 (176 LUAD samples), and GSE30219 (293 LUAD samples). Since each dataset used different genetic chips and algorithms, the cut-off value to divide patients into high or low expression group was based on the median value of NRAS expression in each dataset. As shown in Fig. S1 (GSE50081: $p=0.028$, GSE42127: $p=0.0078$, GSE30219: $p=0.0026$ ), we performed Kaplan-Meier survival analyses as a validation. Hence, NRAS expression level could be an independent prognostic factor for patients with LUAD.

\subsection{GSEA identified NRAS-related signaling pathways}

To further study the signaling pathways that NRAS might be involved in, we performed a GSEA between high and low NRAS expression phenotypes. The gene sets were enriched and analyzed by Hallmark (h.all.v6.2.symbols.gmt) and Go (c5.bp.v6.2.symbols.gmt) Collection.

11 Hallmark items (Fig. 4A) including G2M checkpoint, E2F targets, DNA repair, P13K AKT MTOR signaling, MTORC1 signaling pathways were significantly enriched in NRAS high expression phenotype. According to the enrichment of Go Collection (Fig. 4B and Fig. 4C), high NRAS expression gene sets were significantly correlated with 12 immune -related signaling pathways such as activation of innate immune response, negative regulation of $B$ cell proliferation and $T$ cell regulation signaling pathway. There is no 
signal pathways with low expression in NRAS enriched in neither GO nor Hallmark Collection based on NES, NOM P value, and FDR q value.

\subsection{Correlation analysis between NRAS and TIM in LUAD}

We estimated the relationship between the abundance of TIICs and NRAS expression via the TIMER algorithm. The results shown in Fig. 4A indicates that the expression of NRAS was positively correlated with CD8+ T cells (cor=0.27, $p=1.06 \mathrm{e}-09$ ), macrophages (cor $=0.331, p=4.06 \mathrm{e}-14$ ), and neutrophils (cor $=0.373, p=8.81 e-18$ ), while it was negatively correlated with $B$ cells (cor $=-0.216, p=1.31 e-0.6)$ and CD $4+T$ cells (cor $=-0.108, p=1.65 e-02$ ). In addition, we analyzed the correlation between immune-related biomarkers and NRAS expression. As shown in Fig. 4B, NRAS expression was positively correlated with CD274 (rho=0.333, p=8.59e-15), HAVCR2 (rho=0.239, p=4.01e-08), PDCD1 (rho=0.101, p=2.16e-02), CD47 (rho=0.189, $p=1.64 \mathrm{e}-05)$ and CD24 (rho=0.227, $\mathrm{p}=1.82 \mathrm{e}-07$ ). The results shown in Fig. 4C-E validated the correlation between NRAS and immune-related biomarkers at the protein level via IHC staining of TMAs. Analyzing the IRS score of these immune-related molecules between the NRAS strong staining group and weak staining group, we found that the IRS of PD-L1 ( $p=6.24 \mathrm{e}-3)$, PD-1 ( $p=1.37 \mathrm{e} 04)$, and TIM-3 ( $p=2.47 \mathrm{e}-$ $10)$ were significantly higher in NRAS strong staining group than weak group. The representative stainings were performed in Fig. S2.

\section{Discussion}

NRAS, a gene identified in neuroblastoma and fibrosarcoma cell lines, encodes GTPases involved in cell cycle, proliferation, and differentiation. (Shimizu et al., 1983)As a member of the RAS gene family, similar with KRAS, NRAS plays an essential role in tumorigenesis and progression in various kinds of neoplasms. At present, KRAS has been proved strongly as a significant biomarker in the prognosis of NSCLC, (Ma et al., 2020) while the relationship between NRAS and lung cancer remains unclear. Thus far, only one multiinstitutional study has initially explored the correlation between NRAS mutation and NSCLC. (Ohashi et al., 2013)The potential prognostic value of NRAS for LUAD had not been evaluated due to lack of NRASmutated patients. Hence, we performed a first-ever analysis on the NRAS expression in a large cohort of LUAD based on bioinformatics analysis and real world data. We sought to determine the relationship between NRAS and LUAD, especially as a prognostic biomarker in LUAD. Besides, we tried to screen NRAS-related signaling pathways, TIIC levels and immune biomarkers in order to seek out the potential molecular mechanisms involved in the regulation of LUAD progression and TIM by NRAS.

At present, several studies have shown that the NRAS expression level is associated with the progression of lung cancer via in vivo and in vitro experiments. Giannou et al performed RNA interference and RNA overexpression to modulate NRAS expression level in LUAD cell lines and mouse models, and found that NRAS expression was closely related to LUAD metastasis. (Giannou et al., 2017) Liu et al found targeting NRAS was associated with cisplatin sensitivity in the treatment of lung cancer cell lines. In this study, we provided clinicopathological evidence supporting the role of NRAS expression in development and progression of LUAD. (Liu et al., 2018) We discovered NRAS expression up-regulated in LUAD tissues. 
High NRAS expression group was positively correlated with advanced clinical stage and positive lymph nodes, and caused a worse prognosis both at RNA level and protein level.

Exploring the molecular mechanisms of NRAS in LUAD is beneficial for further investigation of the novel targeted therapy approach. The enrichment analysis of Hallmark collection showed protein secretion, $\mathrm{PI} 3 \mathrm{~K} / \mathrm{Akt} / \mathrm{mTOR}$ signaling, MYC targets, mTORC1 signaling, G2/M checkpoint, E2F targets and DNA repairing pathways were significantly enriched in high expression phenotype. G2/M checkpoint, E2F transcription factor and DNA repairing pathways were closely related to the cell cycle, proliferation and apoptosis. The gene imbalance in these pathways might induce chemotherapy resistance and influence drug sensitivity. Numerous studies reported the potential advantages of cell cycle inhibitors as a group of effective anticancer agents in lung cancer. Whether NRAS could be used as a biomarker for the application of cell cycle inhibitors required more experiments to validate. PI3K/Akt/mTOR, MYC, and mTORC1 signaling pathways were closely related to RAS/RAF cascade. Whitwam et al. reported that NRAS prevented phosphorylation of MYC through PI3K/AKT/mTOR signaling pathway, leading to enhanced activity of endogenous MYC protein. (Whitwam et al., 2007) Song et al. found that targeting NRAS-regulated PI3K/AKT/mTOR cascade, including RAF, MEK, and ERK, by MEK inhibitors could delay tumor growth and angiogenesis in lung cancer. (Song, Liu, \& Zhang, 2017)Thus, the enrichment of cancer-related pathways indicated that NRAS might be a potential target for personalized therapy of LUAD.

According to the enrichment of GO collection, we discovered that NRAS expression is associated with TIM. High expression level of NRAS is significantly correlated with 12 immune -related signaling pathways. To further study the relationship between NRAS expression and TIM, we estimated the abundance of TIIC level via the TIMER algorithm. NRAS expression was negatively correlated with the infiltration level of $B$ cell and CD $4+T$ cell and positively related that of CD8 $+T$ cell, macrophage and neutrophil in LUAD. The GSEA performed on GO collections indicated that high NRAS expression was enriched in activation of innate immune response, negative regulation of $B$ cell proliferation and $T$ cell regulation signaling pathway, which was consistent with the results of TIIC analysis. Brambilla et al. found lower CD4+/CD8 + ratio conferred a worse survival in non-small cell lung cancer.(Brambilla et al., 2016) In addition, multiple studies had proven that $C D 20+B$ cell was associated with improved survival. (Al-Shibli et al., 2008; Pelletier, Edwardes, Michel, Halwani, \& Morin, 2001)Prognostic role of tumor associated macrophages in lung cancer had still been controversial. (Chen et al., 2003; Ho et al., 2008; Kim et al., 2008; Zeni et al., 2007) Besides, by comparing the relationship between NRAS and immunerelated functional molecules, we found NRAS expression was positively correlated with CD274, HAVCR2, PDCD1, CD47 and CD24. CD274 encodes PD-L1, HAVCR2 encodes TIM-3, and PDCD1 encodes PD1. (Dong, Zhu, Tamada, \& Chen, 1999; Ishida, Agata, Shibahara, \& Honjo, 1992; McIntire et al., 2001) We also performed IHC staining in primary LUAD tissues from 325 patients to validate the correlation between NRAS and these immune-related biomarkers. The close relationship between NRAS expression and the immunosuppressive molecules indicated that NRAS might be involved in immune evasion and suppression of immune function in LUAD. Both CD47 and CD24 have been proven as 'don't eat me' signals, which are expressed by tumor-associated macrophages. Cancer cells are expertise in evading 
clearance by macrophages via the overexpression of such anti-phagocytic molecular as CD47 and CD24. (Barkal et al., 2019; Willingham et al., 2012)Thus, the positive correlation between NRAS and 'don't eat me' signals indicates that NRAS might influence phagocytosis of tumor-associated macrophages. From the above results, we found that the expression level of NRAS might indeed cause a poor prognosis of LUAD patients by remodeling the immune microenvironment of a tumor.

There are still several limitations in our study. First, this study is based on the specimens from one institution and bioinformatic analysis from public RNA-seq databases. By the virtue of its retrospective design, our study has a bias by the virtue of potential heterogeneity. Thus, more prospective large-scale clinical studies are required for us to further study the relationship between NRAS and LUAD. Also, the clinicopathological information is not intact from public databases. For instance, tumor size, pathological subtype of LUAD and treatment details are not provided from TCGA and GEO databases.

\section{Conclusions}

To conclude, we have found NRAS expression as a novel prognostic biomarker in LUAD. Besides, we have shown that the expression level of NRAS may influence the prognosis of LUAD via a variety of cancerrelated pathways and TIM remodeling.

\section{Declarations}

Funding: This work was supported by the National Natural Science Foundation of China (grants 81930073 and 81772466), Shanghai Shenkang Hospital Development Center City Hospital Emerging Cutting-edge Technology Joint Research Project (SHDC12017102) and Shanghai Municipal Health Commission Key Discipline Project (2017ZZ02025 and 2017ZZ01019).

Conflicts of interest/Competing interest: Not applicable

Availability of data and material: Publicly available datasets were analyzed in this study, these can be found in The Cancer Genome Atlas (https: //portal.gdc. cancer.gov/), the NCBI Gene Expression Omnibus (GSE30219, GSE42127, and GSE50081), and the Human Protein Atlas(http://www.proteinatlas.org/).

Code availability: Not applicable

Ethics approval: Not applicable

Consent to participate: Not applicable

Consent for publication: Not applicable

\section{References}


1. Al-Shibli KI, Donnem T, Al-Saad S, Persson M, Bremnes RM, Busund LT (2008) Prognostic effect of epithelial and stromal lymphocyte infiltration in non-small cell lung cancer. Clin Cancer Res 14(16):5220-5227. doi:10.1158/1078-0432.Ccr-08-0133

2. Banys-Paluchowski M, Milde-Langosch $K$, Fehm T, Witzel I, Oliveira-Ferrer L, Schmalfeldt B, Müller V (2020) Clinical relevance of H-RAS, K-RAS, and N-RAS mRNA expression in primary breast cancer patients. Breast Cancer Res Treat 179(2):403-414. doi:10.1007/s10549-019-05474-8

3. Barkal AA, Brewer RE, Markovic M, Kowarsky M, Barkal SA, Zaro BW,.. . Weissman IL (2019) CD24 signalling through macrophage Siglec-10 is a target for cancer immunotherapy. Nature 572(7769):392-396. doi:10.1038/s41586-019-1456-0

4. Bertoli E, Giavarra M, Vitale MG, Minisini AM (2019) Neuroblastoma rat sarcoma mutated melanoma: That's what we got so far. Pigment Cell Melanoma Res 32(6):744-752. doi:10.1111/pcmr.12819

5. Brambilla E, Le Teuff G, Marguet S, Lantuejoul S, Dunant A, Graziano S,.. . Tsao MS (2016) Prognostic Effect of Tumor Lymphocytic Infiltration in Resectable Non-Small-Cell Lung Cancer. J Clin Oncol 34(11):1223-1230. doi:10.1200/jco.2015.63.0970

6. Bray F, Ferlay J, Soerjomataram I, Siegel RL, Torre LA, Jemal A (2018) Global cancer statistics 2018: GLOBOCAN estimates of incidence and mortality worldwide for 36 cancers in 185 countries. CA Cancer J Clin 68(6):394-424. doi:10.3322/caac.21492

7. Chen JJ, Yao PL, Yuan A, Hong TM, Shun CT, Kuo ML,.. . Yang PC (2003) Up-regulation of tumor interleukin-8 expression by infiltrating macrophages: its correlation with tumor angiogenesis and patient survival in non-small cell lung cancer. Clin Cancer Res 9(2):729-737

8. Dietrich P, Gaza A, Wormser L, Fritz V, Hellerbrand C, Bosserhoff AK (2019) Neuroblastoma RAS Viral Oncogene Homolog (NRAS) Is a Novel Prognostic Marker and Contributes to Sorafenib Resistance in Hepatocellular Carcinoma. Neoplasia 21(3):257-268. doi:10.1016/j.neo.2018.11.011

9. Dong H, Zhu G, Tamada K, Chen L (1999) B7-H1, a third member of the B7 family, co-stimulates T-cell proliferation and interleukin-10 secretion. Nat Med 5(12):1365-1369. doi:10.1038/70932

10. Downward J (2003) Targeting RAS signalling pathways in cancer therapy. Nat Rev Cancer 3(1):1122. doi: $10.1038 / \mathrm{nrc} 969$

11. Fu F, Zhang Y, Gao Z, Zhao Y, Wen Z, Han H,.. . Chen H (2021) Combination of CD47 and CD68 expression predicts survival in eastern-Asian patients with non-small cell lung cancer. $\mathrm{J}$ Cancer Res Clin Oncol 147(3):739-747. doi:10.1007/s00432-020-03477-3

12. Giannou AD, Marazioti A, Kanellakis NI, Giopanou I, Lilis I, Zazara DE,.. . Stathopoulos GT (2017) NRAS destines tumor cells to the lungs. EMBO Mol Med 9(5):672-686. doi:10.15252/emmm.201606978

13. Ho CC, Liao WY, Wang CY, Lu YH, Huang HY, Chen HY,.. . Yang PC (2008) TREM-1 expression in tumor-associated macrophages and clinical outcome in lung cancer. Am J Respir Crit Care Med 177(7):763-770. doi:10.1164/rccm.200704-6410C

14. Hu Y, Tao SY, Deng JM, Hou ZK, Liang JQ, Huang QG,.. . Liu H (2018) Prognostic Value of NRAS Gene for Survival of Colorectal Cancer Patients: A Systematic Review and Meta-Analysis. Asian Pac J 
Cancer Prev 19(11):3001-3008. doi:10.31557/apjcp.2018.19.11.3001

15. Ishida Y, Agata Y, Shibahara K, Honjo T (1992) Induced expression of PD-1, a novel member of the immunoglobulin gene superfamily, upon programmed cell death. Embo j 11(11):3887-3895

16. Kim DW, Min HS, Lee KH, Kim YJ, Oh DY, Jeon YK,... Heo DS (2008) High tumour islet macrophage infiltration correlates with improved patient survival but not with EGFR mutations, gene copy number or protein expression in resected non-small cell lung cancer. Br J Cancer 98(6):1118-1124. doi:10.1038/sj.bjc. 6604256

17. Li T, Fu J, Zeng Z, Cohen D, Li J, Chen Q,.. . Liu XS (2020) TIMER2.0 for analysis of tumor-infiltrating immune cells. Nucleic Acids Res. doi:10.1093/nar/gkaa407

18. Liloglou T, Bediaga NG, Brown BR, Field JK, Davies MP (2014) Epigenetic biomarkers in lung cancer. Cancer Lett 342(2):200-212. doi:10.1016/j.canlet.2012.04.018

19. Liu X, Lv X, Yang Q, Jin H, Zhou W, Fan Q (2018) MicroRNA-29a Functions as a Tumor Suppressor and Increases Cisplatin Sensitivity by Targeting NRAS in Lung Cancer. Technol Cancer Res Treat 17:1533033818758905. doi:10.1177/1533033818758905

20. Ma Z, Zhang Y, Deng C, Fu F, Deng L, Li Y, Chen H (2020) The prognostic value of Kirsten rat sarcoma viral oncogene homolog mutations in resected lung adenocarcinoma differs according to clinical features. The Journal of Thoracic Cardiovascular Surgery. doi:https://doi.org/10.1016/j.jtcvs.2020.05.097

21. McIntire JJ, Umetsu SE, Akbari O, Potter M, Kuchroo VK, Barsh GS,.. . DeKruyff RH (2001) Identification of Tapr (an airway hyperreactivity regulatory locus) and the linked Tim gene family. Nat Immunol 2(12):1109-1116. doi:10.1038/ni739

22. Ohashi K, Sequist LV, Arcila ME, Lovly CM, Chen X, Rudin CM,.. . Pao W (2013) Characteristics of lung cancers harboring NRAS mutations. Clin Cancer Res 19(9):2584-2591. doi:10.1158/1078-0432.Ccr12-3173

23. Pelletier MP, Edwardes MD, Michel RP, Halwani F, Morin JE (2001) Prognostic markers in resectable non-small cell lung cancer: a multivariate analysis. Can J Surg 44(3):180-188

24. Pérez de Castro I, Diaz R, Malumbres M, Hernández MI, Jagirdar J, Jiménez M,.. . Pellicer A (2003) Mice deficient for $\mathrm{N}$-ras: impaired antiviral immune response and T-cell function. Cancer Res 63(7):1615-1622

25. Shimizu K, Goldfarb M, Suard Y, Perucho M, Li Y, Kamata T,.. . Wigler MH (1983) Three human transforming genes are related to the viral ras oncogenes. Proc Natl Acad Sci U S A 80(8):21122116. doi:10.1073/pnas.80.8.2112

26. Siegel RL, Miller KD, Jemal A (2017) Cancer Statistics, 2017. CA Cancer J Clin 67(1):7-30. doi:10.3322/caac. 21387

27. Song Z, Liu F, Zhang J (2017) Targeting NRAS(Q61K) mutant delays tumor growth and angiogenesis in non-small cell lung cancer. Am J Cancer Res 7(4):831-844

28. Subramanian A, Kuehn H, Gould J, Tamayo P, Mesirov JP (2007) GSEA-P: a desktop application for Gene Set Enrichment Analysis. Bioinformatics 23(23):3251-3253. 
doi:10.1093/bioinformatics/btm369

29. Subramanian A, Tamayo P, Mootha VK, Mukherjee S, Ebert BL, Gillette MA,.. . Mesirov JP (2005) Gene set enrichment analysis: a knowledge-based approach for interpreting genome-wide expression profiles. Proc Natl Acad Sci U S A 102(43):15545-15550. doi:10.1073/pnas.0506580102

30. Suzuki K, Kachala SS, Kadota K, Shen R, Mo Q, Beer DG,... Adusumilli PS (2011) Prognostic immune markers in non-small cell lung cancer. Clin Cancer Res 17(16):5247-5256. doi:10.1158/10780432.Ccr-10-2805

31. Thomas NE, Edmiston SN, Alexander A, Groben PA, Parrish E, Kricker A,.. . Conway K (2015) Association Between NRAS and BRAF Mutational Status and Melanoma-Specific Survival Among Patients With Higher-Risk Primary Melanoma. JAMA Oncol 1(3):359-368.

doi:10.1001/jamaoncol.2015.0493

32. Uhlén M, Fagerberg L, Hallström BM, Lindskog C, Oksvold P, Mardinoglu A,.. . Pontén F (2015) Proteomics. Tissue-based map of the human proteome. Science 347(6220):1260419. doi:10.1126/science.1260419

33. Vogelstein B, Papadopoulos N, Velculescu VE, Zhou S, Diaz LA Jr, Kinzler KW (2013) Cancer genome landscapes. Science 339(6127):1546-1558. doi:10.1126/science.1235122

34. Whitwam T, Vanbrocklin MW, Russo ME, Haak PT, Bilgili D, Resau JH,.. . Holmen SL (2007) Differential oncogenic potential of activated RAS isoforms in melanocytes. Oncogene 26(31):45634570. doi:10.1038/sj.onc. 1210239

35. Willingham SB, Volkmer JP, Gentles AJ, Sahoo D, Dalerba P, Mitra SS,... Weissman IL (2012) The CD47-signal regulatory protein alpha (SIRPa) interaction is a therapeutic target for human solid tumors. Proc Natl Acad Sci U S A 109(17):6662-6667. doi:10.1073/pnas.1121623109

36. Zeni E, Mazzetti L, Miotto D, Lo Cascio N, Maestrelli P, Querzoli P,.. . Boschetto P (2007) Macrophage expression of interleukin-10 is a prognostic factor in nonsmall cell lung cancer. Eur Respir $\mathrm{J}$ 30(4):627-632. doi:10.1183/09031936.00129306

\section{Tables}


Table1. Demographics of including patients in TCGA corhort and FUSCC corhort

\begin{tabular}{|c|c|c|c|}
\hline \multicolumn{2}{|c|}{ Clinical characteristics } & TCGA' corhort $(n=316)$ & FUSCC $\ddagger$ corhort $(n=325)$ \\
\hline \multicolumn{2}{|l|}{ Age } & $64.58 \pm 10.35$ & $60.65 \pm 10.25$ \\
\hline \multirow[t]{2}{*}{ Gender } & Female & $162(51.27 \%)$ & $185(56.92 \%)$ \\
\hline & Male & 154(48.73\%) & $140(43.08 \%)$ \\
\hline \multicolumn{2}{|c|}{ Pack years smoked } & $30.39 \pm 30.70$ & $8.61 \pm 19.47$ \\
\hline \multirow[t]{4}{*}{ Stage } & I & $164(51.90 \%)$ & $251(77.23 \%)$ \\
\hline & II & $75(23.73 \%)$ & $56(17.23 \%)$ \\
\hline & III & $56(17.72 \%)$ & $15(4.62 \%)$ \\
\hline & IV & $21(6.65 \%)$ & $3(0.92 \%)$ \\
\hline \multirow[t]{4}{*}{$\mathrm{T}$} & $\mathrm{T} 1$ & $96(30.38 \%)$ & $251(77.23 \%)$ \\
\hline & $\mathrm{T} 2$ & $177(56.01 \%)$ & $56(17.23 \%)$ \\
\hline & T3 & $25(7.91 \%)$ & $15(4.62 \%)$ \\
\hline & $\mathrm{T} 4$ & $18(5.70 \%)$ & $3(0.92 \%)$ \\
\hline \multirow[t]{4}{*}{$\mathrm{N}$} & No & $201(63.61 \%)$ & $225(69.23 \%)$ \\
\hline & N1 & $65(20.57 \%)$ & $21(6.46 \% \rrbracket$ \\
\hline & N2 & $48(15.19 \%)$ & $79 \llbracket 24.31 \% \rrbracket$ \\
\hline & N3 & $2(0.63 \%)$ & 0 \\
\hline \multirow[t]{2}{*}{ M } & MO & 295(93.35\%) & $325(100 \%)$ \\
\hline & M1 & $21(6.65 \%)$ & 0 \\
\hline
\end{tabular}

† TCGA: The Cancer Genome Atlas

‡ FDUSCC: Fudan University Shanghai Cancer Center 


\begin{tabular}{|llll|}
\hline \multicolumn{4}{|l}{ Table2. NRAS expression associated with clinical pathological characteristics (Logistic regression) } \\
\hline Clinical characters & Total (N) & Odds ratio in NRAS expression & p-value \\
\hline Cigarette status & 316 & $1.17(0.75-1.81)$ & 0.442 \\
\hline Gender & 316 & $1.16(0.75-1.81)$ & 0.500 \\
\hline Age & 316 & $0.99(0.97-1.01)$ & 0.215 \\
\hline Clinical stage (stage I vs. stage III) & 220 & $\mathbf{1 . 9 0 ( 1 . 1 4 - 3 . 2 1 )}$ & $\mathbf{0 . 0 0 5 9 ^ { * * }}$ \\
\hline Tumor size(T1 vs. T2) & 273 & $1.46(0.88-2.41)$ & 0.141 \\
\hline Lymph nodes (positive vs. negative) & 316 & $\mathbf{2 . 0 0 ( 1 . 2 6 - 3 . 2 0 )}$ & $\mathbf{0 . 0 0 3 7 ^ { * * }}$ \\
\hline Metastasis (M0 vs M1) & 316 & $0.90(0.37-2.20)$ & 0.821 \\
\hline
\end{tabular}


Table3. associations with overall survival and clinicopathologic characteristics in TCGA patients using: A. Univariable Cox regression; B. Multivariable Cox regression

\begin{tabular}{|c|c|c|c|c|c|}
\hline \multicolumn{2}{|c|}{ Clinical Characters } & \multirow{2}{*}{$\begin{array}{l}\mathrm{HR}^{\dagger} \\
1.002\end{array}$} & \multirow{2}{*}{$\begin{array}{l}\text { HR.95L } \\
0.984\end{array}$} & \multirow{2}{*}{$\begin{array}{l}\text { HR.95H } \\
1.020\end{array}$} & \multirow{2}{*}{$\begin{array}{l}\text { P value } \\
0.823\end{array}$} \\
\hline A & Age & & & & \\
\hline & Gender & 1.10 & 0.782 & 1.56 & 0.574 \\
\hline & Pack years smoked & 0.999 & 0.993 & 1.01 & 0.664 \\
\hline & $\mathrm{T}$ & 1.58 & 1.30 & 1.93 & $4.51 E-06^{\star \star *}$ \\
\hline & $\mathbf{N}$ & 1.70 & 1.40 & 2.06 & $9.40 \mathrm{E}-08^{\star \star *}$ \\
\hline & $M$ & 1.87 & 1.06 & 3.34 & $0.0317^{*}$ \\
\hline & NRAS & 1.40 & 1.07 & 1.83 & $0.0129^{*}$ \\
\hline \multirow[t]{7}{*}{ B } & age & 1.014 & 0.99 & 1.03 & 0.19 \\
\hline & gender & 0.891 & 0.61 & 1.31 & 0.56 \\
\hline & Pack years smoked & 0.997 & 0.99 & 1.00 & 0.40 \\
\hline & $\mathbf{T}$ & 1.39 & 1.09 & 1.77 & $0.0077^{\star *}$ \\
\hline & $\mathbf{N}$ & 1.47 & 1.15 & 1.87 & $0.0019^{* *}$ \\
\hline & $M$ & 1.68 & 0.88 & 3.19 & 0.11 \\
\hline & NRAS & 1.38 & 1.01 & 1.87 & $0.043^{*}$ \\
\hline
\end{tabular}

†HR: Hazard Ratio

Figures 


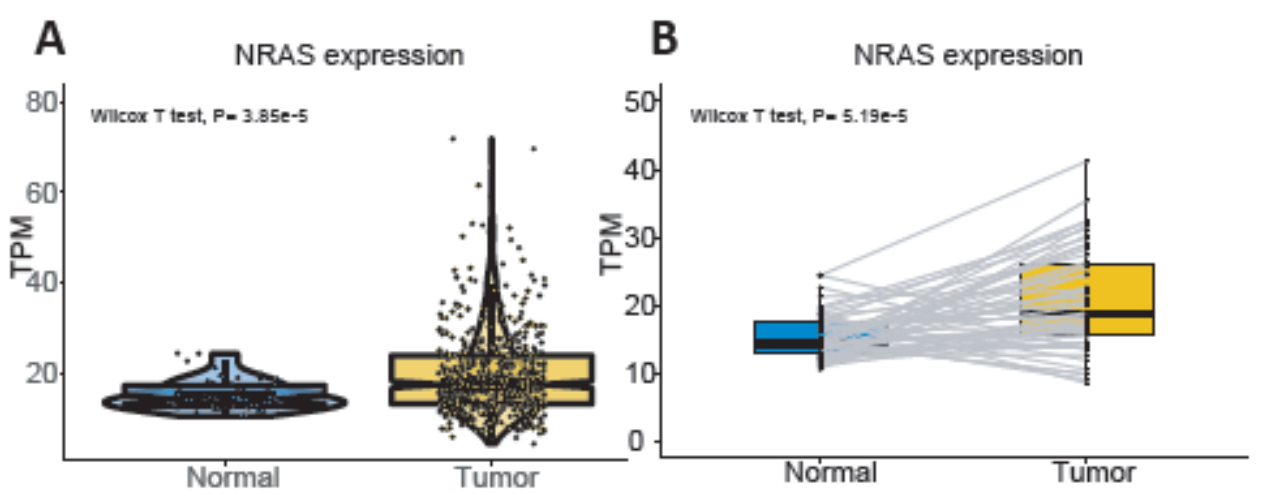

$\epsilon$

D
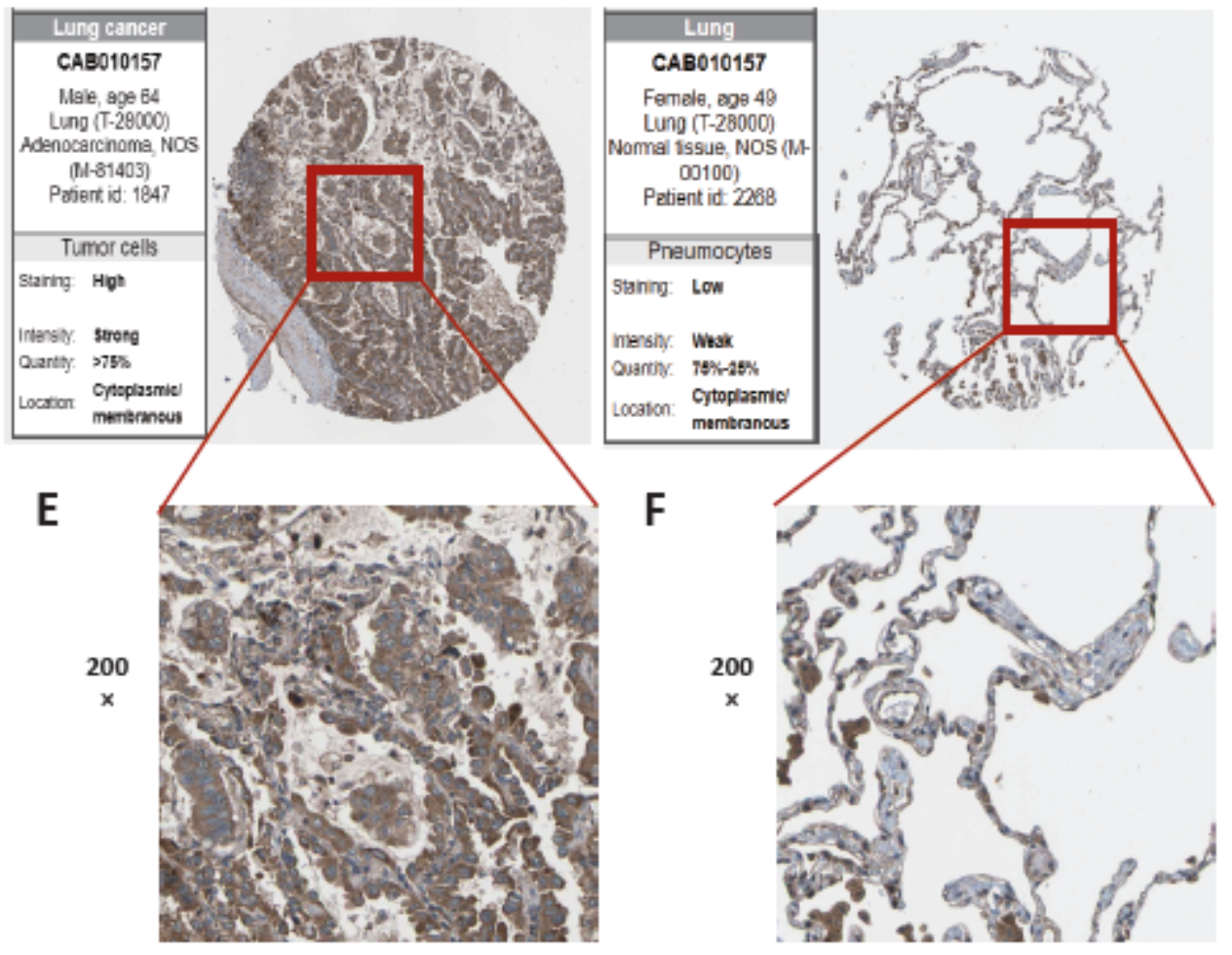

Figure 1

Lung adenocarcinoma (LUAD) highly expressing NRAS. A. The expression of NRAS was significantly upregulated in LUAD compared with normal cells. B. The pair analysis indicated that NRAS expression was significantly upregulated in LUAD. C. Representative Immunohistochemical (IHC) staining for NRAS in LUAD tissue was performed. D. Representative IHC staining for NRAS in normal tissue was performed. E. IHC (200x) staining for NRAS in LUAD tissue F. IHC (200x) staining for NRAS in normal tissue. 

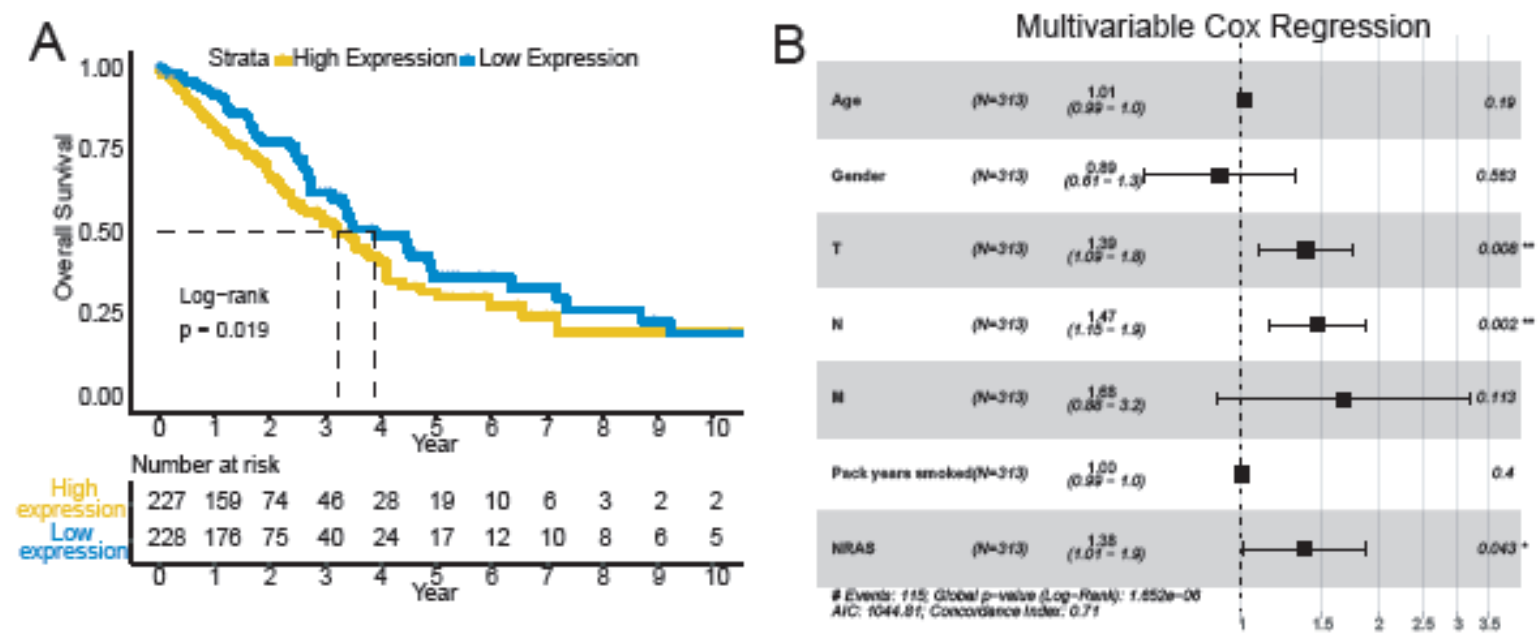

C
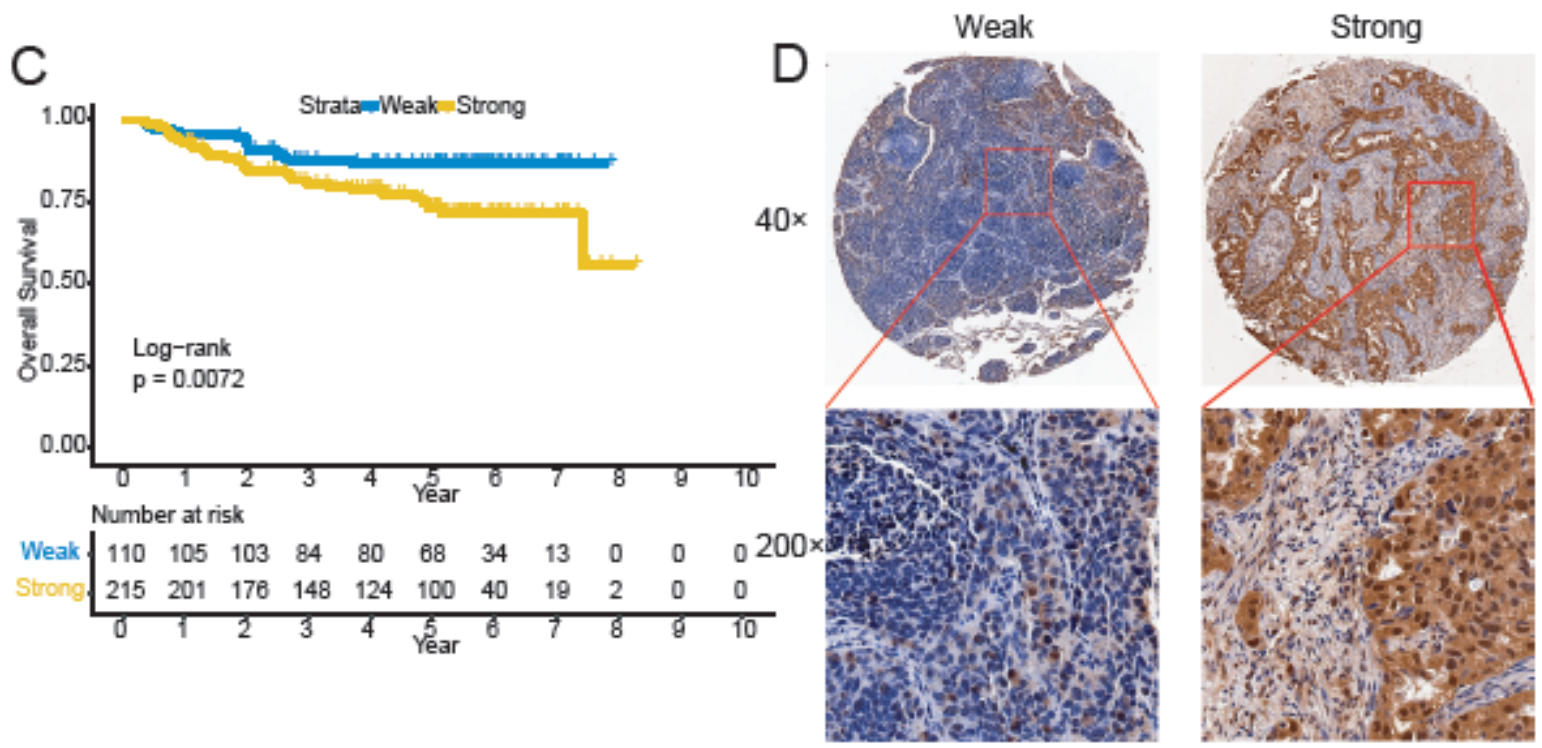

Figure 2

NRAS is associated with poor prognosis in LUAD patients at RNA and protein level. A. Kaplan-Meier curve to compare the overall survival of LUAD patients between high and low NRAS expression group based on TCGA databases. B. Multivariable Cox analysis of NRAS expression and other clinicopathological factors for LUAD patients. C. Kaplan-Meier Curve of overall survival according to protein expression of NRAS in patients with LUAD. D. Representative images of IHC for strong and weak staining of NRAS in LUAD specimens. The cutoff for the IRS score of NRAS was 6. 


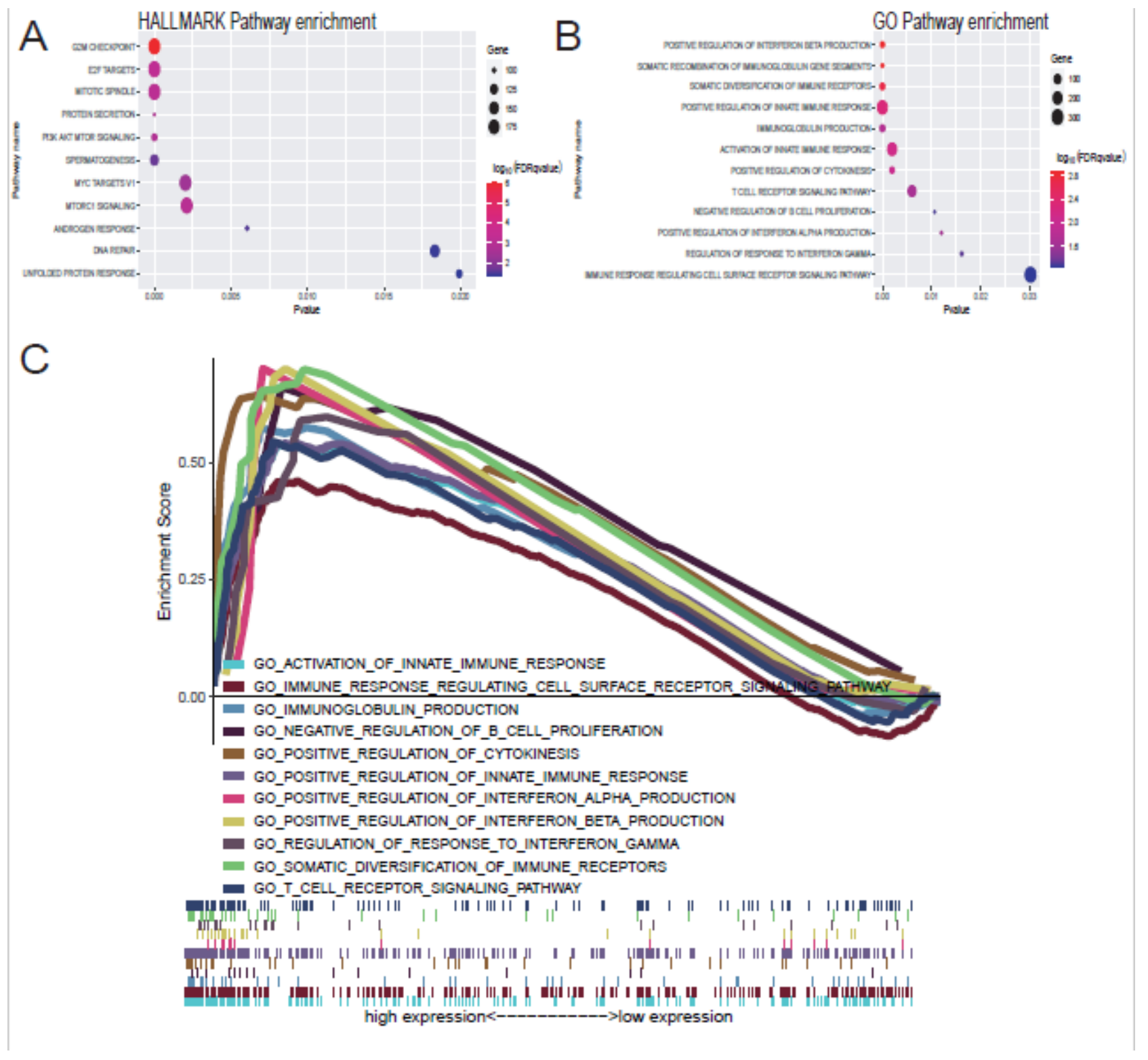

Figure 3

A. 11 signaling pathways including G2M checkpoint, E2F targets, MTORC1 signaling, PI3K-AKT-MTOR signaling, MYC Targets V1, and DNA repair were enriched in Hallmark collection B. 12 signaling pathways including activation of innate immune response, negative regulation of $B$ cell proliferation and $T$ cell regulation signaling pathway were significantly enriched in GO collection. C. A merged enrichment plot from gene set enrichment analysis including enrichment score and gene sets based on GO collection. 

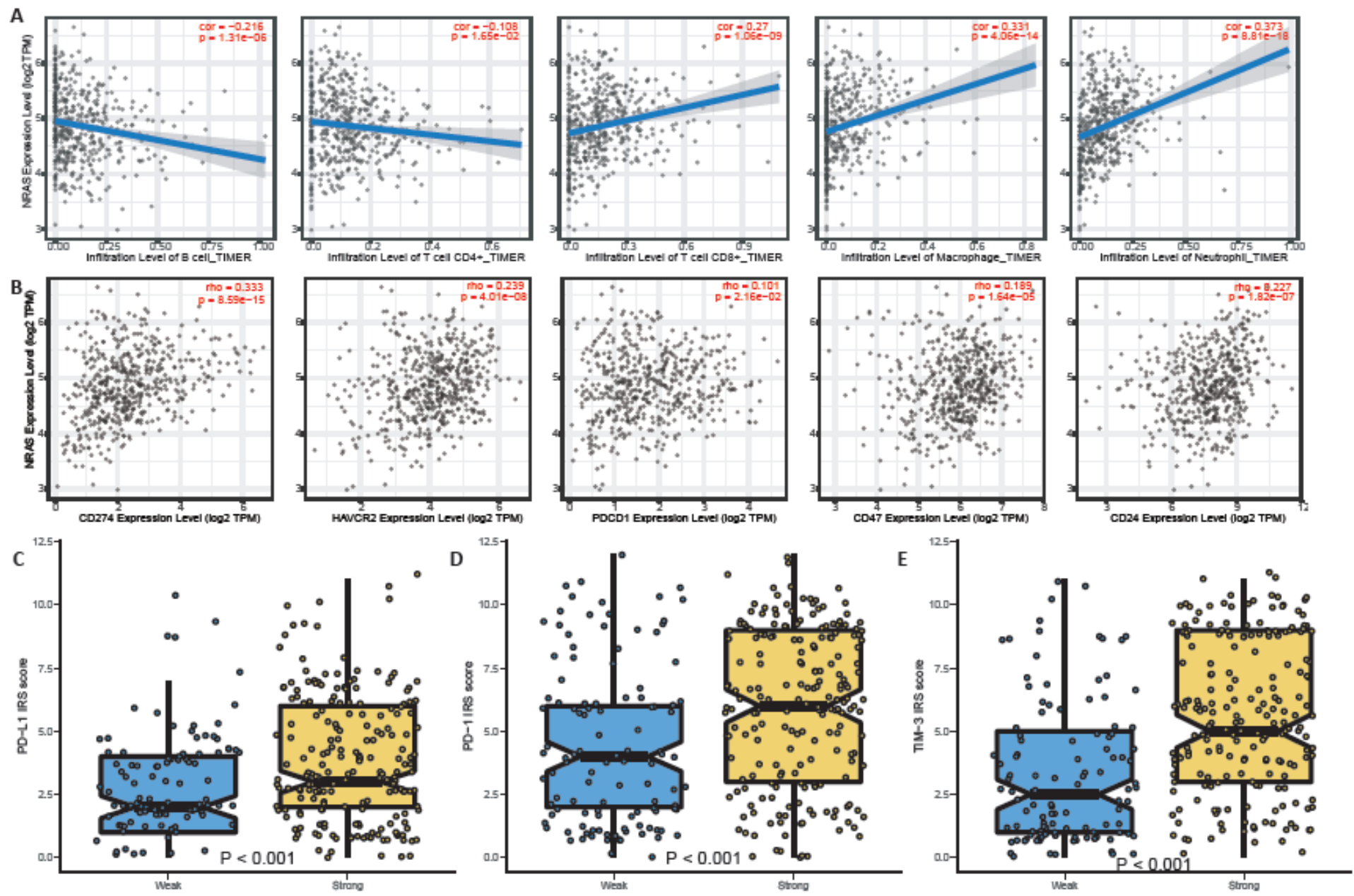

Figure 4

NRAS expression is associated with tumor immune microenvironment. A. NRAS expression level has significant negative correlations with infiltrating levels of CD8+ T cells (cor=0.27, $p=1.06 e-09$ ), macrophages (cor=0.331, $p=4.06 e-14$ ), and neutrophils (cor=0.373, $p=8.81 e-18$ ), while it was negatively correlated with B cells (cor=-0.216, $p=1.31 e-0.6)$ and CD 4+ T cells (cor=-0.108, $p=1.65 e-02)$. B. NRAS expression was positively correlated with CD274 (rho=0.333, $p=8.59 e-15)$, HAVCR2 (rho=0.239, $p=4.01 e-$ 08), PDCD1 (rho=0.101, p=2.16e-02), CD47 (rho=0.189, p=1.64e-05) and CD24 (rho=0.227, p=1.82e-07). C. IRS of PD-L1 was significantly higher in high NRAS expression group at protein level $(p=6.24 e-3)$. D IRS of PD-1 was significantly higher in high NRAS expression group at protein level $(p=1.37 e-04)$. E IRS of TIM-3 was significantly higher in high NRAS expression group at protein level ( $p=2.47 e-10)$.

\section{Supplementary Files}

This is a list of supplementary files associated with this preprint. Click to download.

- SupplementaryMaterial.pdf 